\title{
Acute Respiratory Failure in the Oncologic Patient: New Era, New Issues
}

\author{
B. L. Ferreyro and L. Munshi
}

\subsection{Introduction}

Recent decades have seen an increase in the number of patients living with cancer. This trend has resulted in an increase in intensive care unit (ICU) utilization across this population [1]. Acute respiratory failure is the most frequent medical complication leading to critical illness in oncologic patients [2-4]. Historically, there has been a reluctance to admit cancer patients to the ICU given their poor outcomes, particularly in the setting of hematologic malignancy and invasive mechanical ventilation [5]. ICU treatment limitations or refusal of admission was advocated [6]. Major advances in oncologic care, critical care and more meticulous attention to where the conditions overlap, have resulted in marked improvement in short-term survival in this population $[1,7,8]$. Despite these major advances, acute respiratory failure in this population remains complex with unique challenges surrounding diagnosis and management compared to the general ICU population. This chapter provides a comprehensive overview of acute respiratory failure in the oncologic population and highlights specific considerations for the intensivist. We will focus on the important differences between the immunocompromised oncologic patient and general intensive care population, the spectrum of causes of acute respiratory failure with a specific focus on toxicities related to newer cancer therapies, diagnostic approach, management and an up-to-date overview of prognosis.

\footnotetext{
B. L. Ferreyro

Interdepartmental Division of Critical Care Medicine, Department of Medicine, Mount Sinai Hospital/University Health Network, University of Toronto, Toronto, ON, Canada

Internal Medicine Department, Hospital Italiano de Buenos Aires, Buenos Aires, Argentina

L. Munshi $(\bowtie)$

Interdepartmental Division of Critical Care Medicine, Department of Medicine, Mount Sinai Hospital/University Health Network, University of Toronto, Toronto, ON, Canada

e-mail: Laveena.munshi@ sinaihealthsystem.ca
} 
Table 3.1 Advances in oncologic care and the implications for critical care

\begin{tabular}{l|l}
\hline $\begin{array}{l}\text { Advance } \\
\text { Earlier detection of } \\
\text { disease }\end{array}$ & $\begin{array}{l}\text { New critical care challenges } \\
\text { Earlier treatment with potential toxicities causing acute respiratory } \\
\text { failure }\end{array}$ \\
\hline $\begin{array}{l}\text { Novel and more effective } \\
\text { treatment options }\end{array}$ & $\begin{array}{l}\text { Known/unknown toxicities causing acute respiratory failure with } \\
\text { uncertainty in optimal management (example pulmonary toxicity } \\
\text { due to immune check point inhibitions) }\end{array}$ \\
\hline $\begin{array}{l}\text { Treatments with reduced } \\
\text { toxicities for advanced } \\
\text { age }\end{array}$ & $\begin{array}{l}\text { Older patients with more comorbidities receiving treatment and } \\
\text { admitted to ICU }\end{array}$ \\
\hline Sustained cancer control & Shift from an acute to a chronic disease model \\
\hline
\end{tabular}

\subsection{The Evolution of Oncologic Critical Care over Time}

Historically, candidacy for oncologic treatment was reserved for early stage disease across functional patients with minimal comorbid conditions. Recent advances in oncologic care have resulted in (1) earlier detection of malignancies; (2) a broader range of available therapies across different stages of disease; (3) gentler therapeutic options for the aging population living long enough to be diagnosed with cancer; (4) precision medicine/targeted therapies; and, most recently, (5) engineered T cells that have the ability to re-program the immune system to recognize and attack cancer cells [8-10]. This paradigm shift in cancer care has not been free of new challenges for the medical community. With these advances comes a higher rate of critical illness. While most oncologic admissions to the ICU are planned postsurgical admissions, the profile of those being admitted for medical indications is changing. Infectious acute respiratory failure and sepsis remain the leading causes of medical-oncologic ICU admissions; however, we are seeing a surge in elderly patients presenting with disease- or treatment-associated critical illness and unique toxicities associated with newer therapies. As oncologic care is becoming more complex with intensive regimens, newer therapies and higher volumes, certain centers are developing specialized ICUs for the management of cancer patients, recognizing a need for critical care support and expansion of oncologic critical care knowledge. Table 3.1 highlights some of the recent advances in oncology and the critical care implications of these changes.

\subsection{Differences Between Oncologic and Non-oncologic Patients}

While trying to define the characteristics and outcomes of acute respiratory failure in the oncologic population it is important to consider why this patient population is different to a non-oncologic ICU group. We feel the key differences are associated with (1) their immunocompromised state; (2) higher risk of non-infectious causes of acute respiratory failure; and (3) potential greater risk of frailty at the time of critical 
illness. Oncologic patients are usually considered to be immunocompromised. This can be a result of the underlying disease (e.g., acute leukemia with functional neutropenia) or secondary to the treatment (e.g., patient with solid tumor after chemotherapy). Although the mechanisms leading to immunosuppression are different across all oncologic patients, these patients all share an increased vulnerability to develop common community-acquired and opportunistic infections. Furthermore, the underlying cause of acute respiratory failure in this population can also be noninfectious (direct involvement by the tumor or treatment-related toxicity), making the differential diagnosis even more challenging particularly when infectious/noninfectious causes occur concurrently.

Oncologic patients often exhibit frailty because of their complex trajectory through their disease, exposure to recurrent hospitalizations, risk of nutrition interruption or impairment, and possible exposure to corticosteroids. This state could put them at higher risk in the face of invasive procedures.

It is important to note that the characteristics and outcomes of critically ill oncologic patients differ in those with hematologic malignancies and those with solid tumors. Patients with hematologic malignancies are more frequently admitted with higher acuity conditions, whereas patients with solid tumors are more frequently admitted perioperatively [4]. In the subset of solid tumor patients admitted with medical-induced critical illness, their characteristics and outcomes mirror the general ICU population to a greater degree than those with hematologic malignancies [1]. This difference is attributable to the more profound and prolonged nature of immunosuppression across the hematologic malignancies, making such patients more vulnerable to bacterial, viral and invasive fungal respiratory infections. Furthermore, patients with hematologic malignancies also face a higher risk of noninfectious respiratory complications than those with solid tumors given the heightened doses of cytoreductive/myeloablative therapies, and a higher risk of immune reconstitution reactions.

\subsection{Causes of Acute Respiratory Failure in Oncologic Patients}

Acute respiratory failure in the oncologic population can be broadly classified as infectious and non-infectious. The latter can further be categorized into diseaserelated or treatment-related. The clinician needs to keep a broad differential and always consider the coexistence of more than one cause as is seen in 15-20\% of cases [4]. Table 3.2 outlines the differential diagnoses to consider in the oncologic population.

\subsubsection{Infectious}

Oncologic patients face an increased risk of community-acquired and opportunistic infections, which can be secondary to bacteria, virus and fungi. The lung is a 
Table 3.2 Classification of respiratory failure in oncology patients

\begin{tabular}{|c|c|c|c|}
\hline \multirow{2}{*}{$\begin{array}{l}\text { Predominant } \\
\text { site of } \\
\text { involvement }\end{array}$} & \multirow[b]{2}{*}{ Infectious } & \multicolumn{2}{|l|}{ Non-infectious } \\
\hline & & Disease-related & Treatment-related \\
\hline Airway & & $\begin{array}{l}\text { Extrinsic upper airway } \\
\text { obstruction } \\
\text { Bronchial invasion } \\
\text { Bronchial obstruction }\end{array}$ & $\begin{array}{l}\text { Anaphylactic reactions } \\
\text { to chemotherapy or } \\
\text { targeted therapies }\end{array}$ \\
\hline \multirow[t]{2}{*}{$\begin{array}{l}\text { Lung } \\
\text { parenchyma }\end{array}$} & $\begin{array}{l}\text { Bacterial, fungal, viral } \\
\text { infections }\end{array}$ & $\begin{array}{l}\text { Direct lung invasion by } \\
\text { the tumor } \\
\text { Leukostasis }\end{array}$ & $\begin{array}{l}\text { Cardiogenic pulmonary } \\
\text { edema } \\
\text { Immune checkpoint } \\
\text { Inhibitor toxicity/other } \\
\text { medication-associated } \\
\text { pneumonitis } \\
\text { CAR T-cell induced } \\
\text { lung injury }\end{array}$ \\
\hline & $\begin{array}{l}\text { Acute respiratory } \\
\text { distress syndrome } \\
\text { Pulmonary hemorrhage } \\
\text { (secondary to infection/ } \\
\text { necrotizing pneumonia) }\end{array}$ & $\begin{array}{l}\text { Pulmonary hemorrhage } \\
\text { (secondary to direct } \\
\text { tumor invasion) }\end{array}$ & $\begin{array}{l}\text { Acute respiratory } \\
\text { distress syndrome } \\
\text { Alveolar hemorrhage }\end{array}$ \\
\hline $\begin{array}{l}\text { Vascular } \\
\text { disorders }\end{array}$ & $\begin{array}{l}\text { Angioinvasive } \\
\text { aspergillosis }\end{array}$ & $\begin{array}{l}\text { Thromboembolic disease } \\
\text { Carcinomatous } \\
\text { lymphangitis }\end{array}$ & \\
\hline $\begin{array}{l}\text { Pleura and chest } \\
\text { wall }\end{array}$ & Empyema & $\begin{array}{l}\text { Malignant effusions } \\
\text { Chest wall tumors } \\
\text { Malignant ascites or } \\
\text { bowel obstructions }\end{array}$ & \\
\hline Nervous system & & $\begin{array}{l}\text { Paraneoplastic } \\
\text { neuropathy/myopathy } \\
\text { Spinal cord involvement }\end{array}$ & $\begin{array}{l}\text { Chemotherapy } \\
\text { associated } \\
\text { polyneuropathy }\end{array}$ \\
\hline
\end{tabular}

CAR chimeric antigen receptor

frequent site of infection in patients with cancer. The underlying predominant deficit in the immune system can help narrow the differential of potential pathogens. It is important to note that the clinical presentation of pulmonary infections in this population frequently presents atypical features.

Neutropenic patients face a higher risk of infections caused by staphylococcus species, Gram-negative bacilli and fungal agents, mainly if neutropenia persists. Aspergillus species are the most frequent fungal etiology of pneumonia in neutropenic patients, followed by Zygomycetes and Fusarium.

In patients with impaired cell-mediated immunity, the spectrum of pulmonary infections is different. Intracellular bacteria, such as Listeria, Nocardia and Legionella species, should be considered in this setting. Mycobacterial infectious are also common in patients with cellular immune dysfunction.

Beyond community-acquired viral pathogens, pulmonary viral infections specific to this group include cytomegalovirus (CMV). CMV usually presents diagnostic challenges due to the potential colonization of the airway without established infection. Pneumocystis jirovecii pneumonia is a common opportunistic infection in 
patients with cell-mediated immune dysfunction. Originally described in patients with human immunodeficiency virus (HIV) infection, it has been increasingly recognized as a common pathogen in patients with hematologic malignancies and solid tumors. Recently, Azoulay and colleagues reported the results of a multivariable risk prediction model to assist with the early recognition of $P$. jirovecii pneumonia in patients with hematologic malignancies. The variables in this score included age, lymphoprolipherative disorder, $P$. jirovecii pneumonia prophylaxis, timing of symptoms and ICU admission, the presence of shock and radiological findings [11].

\subsubsection{Non-infectious: Disease-Related}

Disease-induced acute respiratory failure can arise from multiple mechanisms and accounts for approximately $10 \%$ of cases [4]. Direct tumor invasion or extrinsic compression by tumor cells can be seen in carcinomatous lymphangitis, leukostasis, malignant pleural effusions, bulky mediastinal malignancies and tumor cell embolism [2]. Furthermore, pulmonary embolism should always be suspected when assessing the causes of acute respiratory failure in an oncologic patient, particularly in the absence of radiographic evidence of infiltrates.

\subsubsection{Non-infectious: Treatment-Related}

There is a wide range of treatment-associated pulmonary complications. The incidence of treatment-associated lung toxicity across studies evaluating acute respiratory failure in immunocompromised patients is $3.4-10 \%[12,13]$. The predominant mechanism of lung injury stems from an exaggerated inflammatory cytokine release as a consequence of the drug, its impact on the tumor cell, or an appropriate 'on target' but 'off tumor' effect of targeted treatment [9].

\subsubsection{Non-infectious: Treatment-Related-Novel Treatments}

Immune checkpoint inhibitors (ICIs) are a new group of drugs that have revolutionized the treatment of cancer. They work by reprogramming $\mathrm{T}$ cells to generate an anti-tumor response [14-16]. However, they are also associated with inflammatory adverse effects which may range from mild endocrine, dermatologic, and gastrointestinal complications to more severe neurologic or pulmonary toxicity. The incidence of severe lung toxicity associated with ICIs is $2 \%$ [14, 15, 17]. Pneumonitis is considered one of the most common causes of ICI-related death [15]. Pulmonaryrelated toxicity usually presents as pneumonitis at a median time of 3 months posttreatment initiation [18]. Earlier and later time periods have been reported [15]. Obtaining sputum samples for microbiologic assessment or performing bronchoscopy with bronchoalveolar lavage (BAL) can help to exclude concomitant infection. Treatment includes corticosteroids. Immunosuppressants, such as infliximab, cyclophosphamide and mycophenolate, are recommended by some experts in nonrespondent patients although this has not been evaluated extensively $[14,15]$. 
Chimeric antigen receptor- $\mathrm{T}$ cell therapy consists of modified $\mathrm{T}$ cells infused into patients to recognize tumor antigens and initiate an immune attack of cancer cells [9, 16]. Outcomes across Chimeric antigen receptor-T cell therapy intervention trials have been promising for select hematologic malignancies and are projected to revolutionize cancer treatment [10]. Unfortunately, this treatment is associated with cytokine release syndrome, seen in greater than $60 \%$ of cases [9]. Its most severe forms (grade 3-4 toxicity), reported in approximately $15 \%$ of cases, may be associated with acute respiratory failure and acute respiratory distress syndrome (ARDS) [9].

\subsubsection{Non-infectious: Treatment-Related-Tumor Lysis Syndrome}

Tumor lysis syndrome is an acute life-threatening condition resulting from massive release of cell products into the blood $[19,20]$. It is usually associated with rapid cell death after treatment initiation, but it can also occur spontaneously [20]. It is characterized by electrolyte abnormalities (hyperkalemia, hyperphosphatemia, hyperuricemia, hypocalcemia) leading to acute kidney injury, cardiac arrhythmias and potentially multiorgan failure [19]. Life-threatening acute respiratory failure and ARDS associated with tumor lysis syndrome have been described [21, 22].

\subsubsection{Non-infectious: Treatment-Related-Radiation}

Radiation-induced lung injury can present as acute radiation pneumonitis (usually between 1 and 3 months after irradiation) or as pulmonary fibrosis. The acute form usually is self-limiting and responds to corticosteroids whereas the fibrotic form responds poorly to treatment.

\subsubsection{Non-infectious: Treatment-Related-Immune Reconstitution}

Immune recovery or immune reconstitution can lead to acute respiratory failure and is due to an exaggerated inflammatory response as the immune system recovers from a period of prolonged neutropenia. This has been described after neutrophil recovery in hematology malignancy patients or peri-engraftment following autologous/allogeneic stem cell transplant. It is theorized that it may be more common in the setting of an infectious precipitant as is seen in the HIV setting. Treatment includes consideration for corticosteroids depending upon its severity. A series of other inflammatory conditions exist that can cause acute respiratory failure and ARDS, such as inflammation induced by the underlying condition (hemophagocytic lymphohistiocytosis) or treatment (differentiation syndrome from all-trans retinoic acid for acute promyelocytic leukemia) [23]. The allogeneic stem cell transplant patient has a series of unique treatment-associated conditions that can induce acute respiratory failure and severe ARDS. These conditions, range from pre-engraftment diffuse alveolar hemorrhage, to peri-engraftment syndrome and idiopathic pneumonia syndrome [24-26]. While these occur at different phases of the post-stem cell trajectory, the consistent features associated with these conditions include their association with preconditioning regimens and their high mortality in the setting of severe respiratory failure and need for invasive mechanical ventilation. 


\subsection{Diagnostic Work-Up and Undiagnosed Acute Respiratory Failure}

An accurate diagnosis is key to guide early prognostication, institution of appropriate therapy, and initiation of necessary supportive care. Recognizing and projecting the reversibility of the underlying cause is an important factor in deciding the need for monitoring, transfer for ICU admission and eventually the decision for intubation. This also needs to take into consideration the oncologic diagnosis, pending treatments and underlying prognosis. A wide spectrum of differential diagnosis should be kept in consideration, including infectious and non-infectious causes and the possibility of dual infections or concomitant infections/non-infectious etiologies. In patients with pulmonary infiltrates, laboratory studies need to be considered in light of the type and degree of immunosuppression. The work-up may include a series of non-invasive serum and sputum microbiologic tests (sputum cultures, induced sputum for $P$. jirovecii, CMV serum evaluation, serum galactomannan, nasopharyngeal swab for viral polymerase chain reaction, etc.); imaging modalities (computed tomography [CT] thorax, echocardiography if cardiogenic pulmonary edema is considered) and possible BAL for further microbiologic evaluation if no diagnosis has been yielded. The routine and upfront use of BAL in hypoxic oncologic immunocompromised patients with undetermined lung infiltrates is controversial. Conflicting evidence exists surrounding its safety in patients with high oxygen requirements (potentially precipitating endotracheal intubation) [27, 28]; however, evidence has demonstrated that given the debatable diagnostic yield, a strategy of first pursuing non-invasive tests may be warranted in the appropriate population [27]. There may exist a subset of patients for whom the need for BAL as an initial diagnostic strategy is warranted at an earlier time point (e.g., to rule out extrinsic compression or pulmonary hemorrhage, or to rule out infections with potentially toxic treatment profiles). Figure 3.1 outlines an approach to diagnosis.

Finally, despite an extensive diagnostic work up, approximately $13-40 \%$ of oncologic patients have undiagnosed acute respiratory failure [4, 29]. Undiagnosed acute respiratory failure is associated with a worse prognosis. It remains unclear whether it is a disease entity within itself or occult infectious/non-infectious etiology not appropriately identified. The role of lung biopsy has been considered in this population in the setting of acute respiratory failure or ARDS, but, given the immunosuppressed state, frequent thrombocytopenia, risk of bronchopleural fistula in light of positive pressure ventilation, and morbidity and mortality with the procedure; it is not pursued frequently [30].

\subsection{Management}

The mainstays of management of the oncologic patient with acute respiratory failure remain rapid identification of etiology and institution of appropriate therapy. This therapy may range from antimicrobial coverage for possible infections, chemotherapy/radiation therapy for disease-induced acute respiratory failure (e.g., leukostasis 


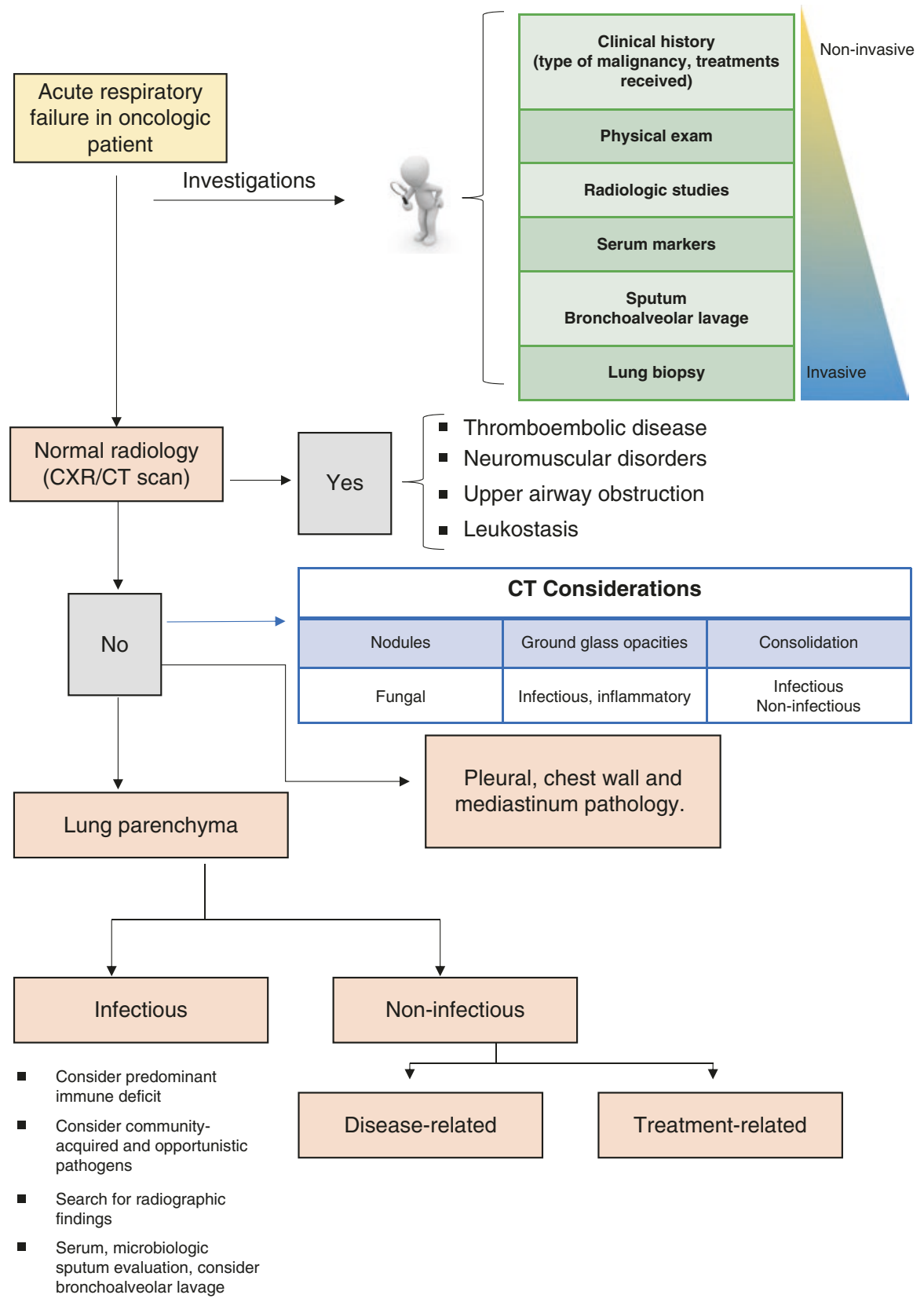

Fig. 3.1 Diagnostic considerations in acute respiratory failure among oncologic patients 
or airway compression from lymphoma), or corticosteroid/anti-interleukin-6 (IL-6) therapy to blunt inflammation related to possible drug toxicities. Of utmost importance is the rapid identification of cause and initiation of treatment early in the course of acute respiratory failure in order to prevent the development of ARDS or need for invasive mechanical ventilation. For disease-associated complications, such as leukostasis or tumor infiltration, historic reservations to initiate chemotherapy in the ICU have been dispelled by more recent data demonstrating its feasibility and lack of association with increased mortality [31]. While mortality rates across acute respiratory failure in this population have decreased $(40-70 \%)[3,7,32]$, there is a subset that continue to have an unacceptably high mortality, particularly in the setting of invasive mechanical ventilation $[32,33]$. The high mortality in the face of mechanical ventilation is likely attributable to (1) more aggressive infectious organisms with a higher risk of drug resistance; (2) adverse events of appropriate antimicrobial treatments; (3) an immunocompromised state that delays eradication of the infection; (4) difficulty or delays in diagnosis; and (5) a higher risk of frailty — which may subject these patients to a higher risk of respiratory muscle weakness prolonging their recovery from critical illness. It remains unclear whether these patients are more susceptible to ventilator-associated lung injury or the harms associated with ventilator-associated lung injury. Given an increased mortality in the setting of invasive mechanical ventilation compared to the general ICU population, a subset of literature has focused on optimal strategies of non-invasive oxygen therapy to prevent intubation.

\subsubsection{Supportive Care During Early Acute Hypoxemic Respiratory Failure}

Three non-invasive oxygen strategies have been evaluated in immunocompromised and oncologic acute respiratory failure: continuous oxygen therapy, non-invasive ventilation, and high-flow nasal cannula (HFNC) — each with unique physiologic properties. They are often applied in the setting of severe hypoxemic acute respiratory failure in an attempt to prevent the need for invasive mechanical ventilation.

Continuous low-flow oxygen therapy via face mask is the most common mechanism of oxygen delivery in the setting of acute hypoxemic respiratory failure. Its greatest limitation remains the limited inspired flow rate (up to $15 \mathrm{~L} / \mathrm{min}$ ). When hypoxia is combined with a high work of breathing, entrainment of ambient air (which could be liters) from around the mask may result in a dilution of the inspired oxygen to the alveoli. This results in insufficient delivery of the intended oxygen concentrations to the alveoli. Inability to provide alveolar recruitment and the risk of local oxygen toxicity (tracheobronchitis/mucociliary disturbance) are also limitations to this non-invasive strategy. 
Non-invasive ventilation (NIV) has the ability to provide continuous positive airway pressure and assist each breath with augmented pressure. The seal generated by the NIV interface (via facemask or helmet) permits alveolar recruitment. Two historic trials in immunocompromised patients established biologic plausibility for the benefit of NIV compared to continuous oxygen therapy in patients with acute respiratory failure $[34,35]$. These trials demonstrated that, in early acute respiratory failure, the application of NIV instead of continuous oxygen therapy was associated with a decreased need for invasive mechanical ventilation and mortality. Given the limited generalizability of those historic trials to the populations we manage today, a more recent trial by Lemiale and colleagues re-evaluated this question and demonstrated no difference in outcomes between NIV and continuous oxygen therapy [36].

Humidified HFNC is a novel device for oxygen delivery that allows for higher flow oxygen delivery via large bore nasal cannula (40-60 L/min of flow). Theoretic mechanisms of benefit include higher flow of inspired oxygen delivery outstripping any ambient air that could be entrained, dead space washout, a small amount of positive end-expiratory pressure (PEEP) generated and humidification to enhance mucociliary clearance. The Florali trial by Frat and colleagues demonstrated reduced 90-day mortality with HFNC compared to NIV and continuous oxygen therapy across the general ICU population with acute respiratory failure [37]. In a post hoc analysis focusing on immunocompromised patients (excluding severe neutropenia), there were no differences with HFNC compared to continuous oxygen on rates of intubation but a persistent benefit of HFNC compared to NIV [38]. These results are intriguing as it is theorized that it may not be the HFNC per se resulting in a beneficial impact, but potentially harm induced by NIV. The median tidal volumes in the NIV arm were $9 \mathrm{~mL} / \mathrm{kg}$ thus leading the authors to hypothesize that ventilator-associated lung injury could be contributing to the increased harm noted. The authors, in a subsequent study, found that a $\mathrm{PaO}_{2} / \mathrm{FiO}_{2}<200 \mathrm{mmHg}$ or tidal volumes $\geq 9 \mathrm{~mL} / \mathrm{kg}$ were associated with NIV failure [39].

Finally, in a systematic review by Sklar and colleagues evaluating non-invasive oxygen strategies in immunocompromised patients, the majority of whom had an oncologic diagnosis, seven studies (randomized controlled trials [RCTs] or observational studies with propensity score matching) were evaluated comparing HFNC to conventional oxygen or NIV. Mortality was found to be lower in the HFNC arms compared to the pooled oxygen control arms (continuous oxygen therapy or NIV). This study was exploratory but warrants further investigation [40].

\subsubsection{Supportive Care During ARDS}

A secondary analysis of the LUNG SAFE database, an international, multicenter observational study of patients with ARDS, was conducted focusing on an immunocompromised cohort [29]. Across this cohort, 20\% of patients with ARDS underwent NIV as their first line supportive management instead of invasive mechanical ventilation. Focusing on observational studies of ARDS across oncology patients, 40-50\% underwent NIV as first-line [32, 33]. While the original studies evaluating the utility of NIV in immunocompromised patients focused on early acute respiratory failure 
and the role of NIV in preventing the need for intubation, these initial promising data has led to NIV creep into more severe subgroups of ARDS [29, 32, 33]. In an observational study by Azoulay and colleagues evaluating management and outcomes of oncologic patients with ARDS, 70\% of those who underwent initial NIV, eventually failed and required invasive mechanical ventilation [32]. However, high failure rates are not consistently reported, as the failure rate noted by Rathi and colleagues was $40 \%$ [33]. Across these two studies-amongst the largest to evaluate ARDS in the oncology population-NIV failure was an independent risk factor for mortality with mortality rates higher than in patients managed with an initial strategy of invasive mechanical ventilation. Given the observational nature of these data, it remains unclear whether this increased mortality is due to unmeasured confounders, such as higher severity of illness not captured in the multivariable analysis, physician reservation to intubate based upon subtle patient factors (e.g., frailty) or whether the use of NIV was associated with higher tidal volumes resulting in greater lung injury and increased mortality in this population. On the contrary, of the $60 \%$ who did not require invasive mechanical ventilation after NIV in the study by Rathi and colleagues, their ICU mortality rates were the lowest (29\%) compared to cohorts with an initial strategy of invasive mechanical ventilation (61\%) and NIV failure (71\%). Given the retrospective nature of these studies, future, research should focus on delineating how to identify which patient populations, if any, may benefit from a time-limited trial of NIV in the setting of ARDS. Until further research, we propose the following management algorithm to consider in oncologic patients in the setting of acute respiratory failure and ARDS (Fig. 3.2).

Acute respiratory distress syndrome (ARDS)

Early acute respiratory failure

High-flow nasal cannula

Continuous oxygen therapy
Mild ARDS

Moderate ARDS

Severe ARDS

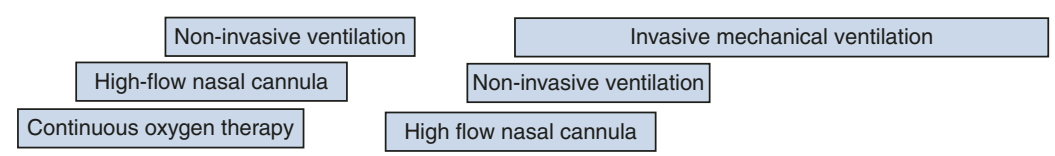

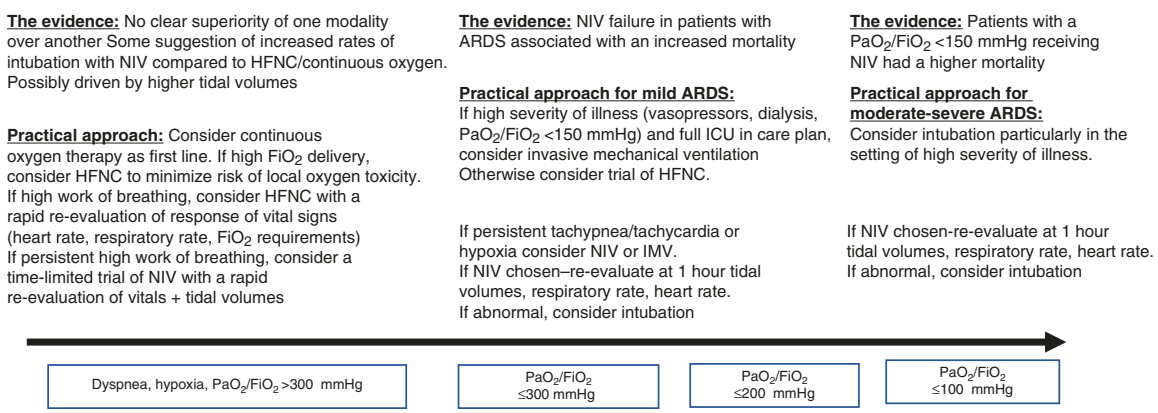

Severity of acute respiratory failure

Fig. 3.2 Invasive and non-invasive oxygenation strategies for oncology patients with acute hypoxemic respiratory failure. NIV non-invasive ventilation, HFNC high-flow nasal cannula; IMV invasive mechanical ventilation 


\subsection{Prognosis and Future Research Considerations}

Historic hospital mortality rates across patients with hematologic malignancy developing critical illness were $>80 \%$ [41]. More recent up-to-date data demonstrate more favorable short-term prognoses ranging from $<10 \%$ in the post-surgical oncologic patient, 30-50\% in the solid or hematologic malignancy patient, to upwards of $60 \%$ across allogeneic stem cell transplant patient requiring invasive mechanical ventilation [42-45]. Improved outcomes are likely attributable to better patient selection for oncologic and intensive care, advances in antimicrobial prophylaxis, infection control and antimicrobial management, earlier identification and a better understanding of the causes of acute respiratory failure and better overall supportive care with more specialized centers/units and subspecialists managing these patients. Factors consistently found to be associated with high mortality in the oncologic critically ill population include age and comorbid conditions, poor functional and frailty status, treatment refractory graft-vs-host disease in allogeneic stem cell transplant patients, NIV failure in the setting of ARDS, invasive fungal infections requiring invasive mechanical ventilation and number of organs failed evaluated after 3-5 days of ICU admission and mechanical ventilation. Early and routine discussions with the oncologist and patient/substitute decision makers in guiding ongoing care decisions is imperative to ensuring that the resources dedicated to the care of this patient is bridging them to a meaningful recovery or candidacy for ongoing oncologic treatment if indicated [46].

Future research should focus on advancing diagnostic strategies to delineate more precisely and rapidly the etiology of acute respiratory failure, strategies to blunt pulmonary and systemic inflammation that can result as a consequence of otherwise effective cancer therapies, the role of biomarkers in predicting the onset of acute respiratory failure, clarifying optimal non-invasive oxygen strategies and the role of NIV and finally long-term outcomes across survivors of acute respiratory failure.

\subsection{Conclusion}

With the paradigm shift in cancer care comes a unique host of critical care conditions and challenges for the intensivist. Acute respiratory failure in the oncologic host has a very broad infectious and non-infectious differential. Early recognition of acute respiratory failure and a thorough evaluation for the etiology is critical to preventing progression to ARDS and need for invasive mechanical ventilation. Optimal non-invasive oxygen strategies remain unclear. Adherence to pressureand volume-limited strategies remains imperative in this population. Future research must focus on better identification of 'undiagnosed' acute respiratory failure, optimal initial supportive care strategies for mild ARDS and long-term outcomes. 


\section{References}

1. Taccone F, Artigas A, Sprung C, et al. Characteristics and outcomes of cancer patients in European ICUs. Crit Care. 2009;13:R15.

2. Pastores SM. Acute respiratory failure in critically ill patients with cancer. Diagnosis and management. Crit Care Clin. 2001;17:623-46.

3. Mokart D, Etienne A, Esterni B, et al. Critically ill cancer patients in the intensive care unit: short-term outcome and 1-year mortality. Acta Anaesthesiol Scand. 2011;56:178-89.

4. Azoulay E, Pickkers P, Soares M, et al. Acute hypoxemic respiratory failure in immunocompromised patients: the Efraim multinational prospective cohort study. Intensive Care Med. 2017;316:1-12.

5. Kress JP, Christenson J, Pohlman AS, et al. Outcomes of critically ill cancer patients in a university hospital setting. Am J Respir Crit Care Med. 1999;160:1957-61.

6. Task Force of the American College of Critical Care Medicine, Society of Critical Care Medicine. Guidelines for intensive care unit admission, discharge and triage. Crit Care Med. 1999;27:633-8.

7. Azoulay E, Mokart D, Pène F, et al. Outcomes of critically ill patients with hematologic malignancies: prospective multicenter data from France and Belgium-a groupe de recherche respiratoire en réanimation onco-hématologique study. J Clin Oncol. 2013;31:2810-22.

8. Schellongowski P, Sperr W, Wohlfarth P, et al. Critically ill patients with cancer: chances and limitations of intensive care medicine-a narrative review. ESMO Open. 2016;201:1.

9. Gutierrez C, McEvoy C, Mead E, et al. Management of the critically ill adult chimeric antigen receptor-t cell therapy patient. Crit Care Med. 2018;46:1402-10.

10. June CH, Sadelain M. Chimeric antigen receptor therapy. N Engl J Med. 2018;379:64-73.

11. Azoulay E, Roux A, Vincent F, et al. A multivariable prediction model for Pneumocystis jirovecii pneumonia in hematology patients with acute respiratory failure. Am J Respir Crit Care Med. 2018;198:1519-26.

12. Dhokarh R, Li G, Schmickl CN, et al. Drug-associated acute lung injury. Chest. 2012;142:845-50.

13. Reinert T, Baldotto CS, Nunes FAP, et al. Bleomycin induced lung injury. J Cancer Res. 2013;2013:1-9.

14. Wills B, Brahmer JR, Naidoo J. Treatment of complications from immune checkpoint inhibition in patients with lung cancer. Curr Treat Options Oncol. 2018;19:1-21.

15. Brahmer JR, Lacchetti C, Schneider BJ, et al. Management of immune-related adverse events in patients treated with immune checkpoint inhibitor therapy: American Society of Clinical Oncology Clinical Practice Guideline. J Clin Oncol. 2018;36:1714-68.

16. Neelapu SS, Tummala S, Kebriaei P, et al. Chimeric antigen receptor T-cell therapy-assessment and management of toxicities. Nat Rev Clin Oncol. 2018;15:47-62.

17. Nishino M, Giobbie-Hurder A, Hatabu H, Ramaiya NH, Hodi FS. Incidence of programmed cell death 1 inhibitor-related pneumonitis in patients with advanced cancer: a systematic review and meta-analysis. JAMA Oncol. 2016;2:1607-16.

18. Postow M, Sidlow R, Hellmann D. Immune related adverse events associated with immune checkpoint blockade. N Engl J Med. 2018;378:158-68.

19. Mirrakhimov AE, Voore P, Khan M, et al. Tumor lysis syndrome: a clinical review. World J Crit Care Med. 2015;4:130-8.

20. Howard SC, Jones DP, Pui CH. The tumor lysis syndrome. N Engl J Med. 2011;364:1844-54.

21. Macaluso A, Genova S, Maringhini S, et al. Acute respiratory distress syndrome associated with tumor lysis syndrome in a child with acute lymphoblastic leukemia. Pediatr Rep. 2015;7:5760-2.

22. Bell CM, Stewart TE. Acute respiratory distress syndrome associated with tumour lysis syndrome in leukemia. Can Respir J. 1997;4:48-51.

23. Seguin A, Galicier L, Boutboul D, et al. Pulmonary involvement in patients with hemophagocytic lymphohistiocytosis. Chest. 2016;149:1294-301. 
24. Yadav H, Nolan ME, Bohman JK, et al. Epidemiology of acute respiratory distress syndrome following hematopoietic stem cell transplantation. Crit Care Med. 2016;44:1082-90.

25. Lucena CM, Torres A, Rovira M, et al. Pulmonary complications in hematopoietic SCT: a prospective study. Bone Marrow Transplant. 2014;49:1293-9.

26. Panoskaltsis-Mortari A, Griese M, Madtes DK, et al. An official American Thoracic Society research statement: noninfectious lung injury after hematopoietic stem cell transplantation: idiopathic pneumonia syndrome. Am J Respir Crit Care Med. 2011;183:1262-79.

27. Azoulay E, Mokart D, Lambert J, et al. Diagnostic strategy for hematology and oncology patients with acute respiratory failure. Am J Respir Crit Care Med. 2010;182:1038-46.

28. Deotare U, Merman E, Pincus D, et al. The utility and safety of flexible bronchoscopy in critically ill acute leukemia patients: a retrospective cohort study. Can J Anaesth. 2018;65:2 $72-9$.

29. Cortegiani A, Madotto F, Gregoretti C, et al. Immunocompromised patients with acute respiratory distress syndrome: a secondary analysis of the LUNG SAFE database. Crit Care. 2018;22:157-72.

30. Chellapandian D, Lehrnbecher T, Phillips B, et al. Bronchoalveolar lavage and lung biopsy in patients with cancer and hematopoietic stem-cell transplantation recipients: a systematic review and meta-analysis. J Clin Oncol. 2015;33:501-15.

31. Pastores S, Goldman D, Shaz D, et al. Characteristics and outcomes of patient with hematologic malignancies receiving chemotherapy in the intensive care unit. Cancer. 2018;124: 3025-36.

32. Azoulay E, Lemiale V, Mokart D, et al. Acute respiratory distress syndrome in patients with malignancies. Intensive Care Med. 2014;40:1106-14.

33. Rathi N, Haque S, Nates R, et al. Noninvasive positive pressure ventilation vs invasive mechanical ventilation as first-line therapy for acute hypoxemic respiratory failure in cancer patients. $\mathrm{J}$ Crit Care. 2017;39:56-62.

34. Hilbert G, Gruson D, Vargas F, et al. Noninvasive ventilation in immunosuppressed patients with pulmonary infiltrates. N Engl J Med. 2001;344:481-7.

35. Antonellli M, Conti G, Bufi M, et al. Noninvasive ventilation for treatment of acute respiratory failure in patients undergoing solid organ transplant: a randomized trial. JAMA. 2000;283:235-41.

36. Lemiale V, Mokart D, Resche-Rigon M, et al. Effect of noninvasive ventilation vs oxygen therapy on mortality among immunocompromised patients with acute respiratory failure: a randomized clinical trial. JAMA. 2015;314:1711-20.

37. Frat JP, Thille AW, Mercat A, et al. High-flow oxygen through nasal cannula in acute hypoxemic respiratory failure. N Engl J Med. 2015;372:2185-96.

38. Frat JP, Ragot S, Girault C, et al. Effect of non-invasive oxygenation strategies in immunocompromised patients with severe acute respiratory failure: a post-hoc analysis of a randomised trial. Lancet Respir Med. 2016;4:646-53.

39. Frat JP, Ragot S, Coudroy R, et al. Predictors of intubation in patients with acute hypoxemic respiratory failure treated with a noninvasive oxygenation strategy. Crit Care Med. 2018;46:208-15.

40. Sklar M, Mohamed A, Orchanian-Cheff A, et al. The impact of high flow nasal oxygen in the immunocompromised critically ill: a systematic review and meta-analysis. Respir Care. 2018; 63:1555-66.

41. Lloyd-Thomas A, Dhaliwal $\mathrm{H}$, Lister $\mathrm{T}$, et al. Intensive therapy for life-threatening medical complications of hematologic malignancy. Intensive Care Med. 1986;12: 317-24.

42. Bos MM, Bakhshi-Raiez F, Dekker JW, et al. Outcomes of intensive care unit admissions after elective cancer surgery. Eur J Surg Oncol. 2013;39:584-92.

43. Fisher R, Dangoisse C, Crichton S, et al. Short-term and medium-term survival of critically ill patients with solid tumors admitted to the intensive care unit: a retrospective analysis. BMJ Open. 2016;6:e11363. 
44. Darmon MR, Bernal T, Borges M, et al. Ventilatory support in critically ill hematology patients with respiratory failure. Crit Care. 2012;16:R133.

45. Van Vliet M, Verburg I, Boogaard M, et al. Trends in admission prevalence, illness severity and survival of hematological patients treated in Dutch intensive care units. Intensive Care Med. 2014;40:1275-84.

46. Soares M, Bozza F, Azevedo L, et al. Effects of organizational characteristics on outcomes and resource use in patients with cancer admitted to Intensive Care Units. J Clin Oncol. 2016;34:3315-24. 